\title{
New Exact Solutions for a Higher-Order Wave Equation of KdV Type Using Extended F-Expansion Method
}

\author{
Yinghui He, Yun-Mei Zhao, and Yao Long \\ Department of Mathematics, Honghe University, Mengzi, Yunnan 661100, China \\ Correspondence should be addressed to Yinghui He; heyinghui07@163.com
}

Received 23 September 2013; Accepted 4 December 2013

Academic Editor: Carlo Cattani

Copyright (C) 2013 Yinghui He et al. This is an open access article distributed under the Creative Commons Attribution License, which permits unrestricted use, distribution, and reproduction in any medium, provided the original work is properly cited.

\begin{abstract}
The F-expansion method is used to find traveling wave solutions to various wave equations. By giving more solutions of the general subequation, an extended F-expansion method is introduced by Emmanuel. In our work, a generalized KdV type equation of neglecting the highest-order infinitesimal term, which is an important water wave model, is discussed by using the extended F-expansion method. And when the parameters satisfy certain relations, some new exact solutions expressed by Jacobi elliptic function, hyperbolic function, and trigonometric function are obtained. The related results are enriched.
\end{abstract}

\section{Introduction}

It has recently become more interesting to obtain exact solutions of nonlinear partial differential equations. These equations are mathematical models of complex physical phenomena that arise in engineering, applied mathematics, chemistry, biology, mechanics, physics, and so forth. Thus, the investigation of the traveling wave solutions to nonlinear evolution equations (NLEEs) plays an important role in mathematical physics. A lot of physical models have supported a wide variety of solitary wave solutions.

In the recent years, much effort has been spent on this task and many significant methods have been established such as inverse scattering transform [1], Backlund and Darboux transform [2], Hirota [3], homogeneous balance method [4], Jacobi elliptic function method [5], tanh-function method [6], exp-function method [7], simple equation method [8], F-expansion method $[9,10]$, improved F-expansion method [11], and extended F-expansion method [12].

Wang and Li [10] developed a new algebraic method, belonging to the simplest equation method [13-16], to seek more new solutions of NLEEs that can be expressed as polynomial in an elementary function which satisfies a more general subequation than other subequations like Riccati equation, auxiliary ordinary equation, elliptic equation, and generalized Riccati equation. The Fans method not only gives a unified formation to construct various traveling wave solutions but also provides a guideline to classify the various types of traveling wave solutions according to five parameters. An extended F-expansion method was proposed by Yomba in 2005 by giving more solutions of the general subequation. Using the new method, exact solutions of many NLEEs are successfully obtained [12].

In this work, we apply the extended F-expansion method on a higher-order wave equation of KdV type for obtaining new exact traveling solutions.

In 1995, based on the physical and asymptotic considerations, Fokas [17] derived the following generalized KdV equation:

$$
\begin{aligned}
\eta_{t} & +\eta_{x}+\alpha \eta \eta_{x}+\beta \eta_{x x x}+\rho_{1} \alpha^{2} \eta^{2} \eta_{x} \\
& +\alpha \beta\left(\rho_{2} \eta \eta_{x x x}+\rho_{3} \eta_{x} \eta_{x x}\right)+\rho_{4} \alpha^{3} \eta^{3} \eta_{x} \\
& +\alpha^{2} \beta\left(\rho_{5} \eta^{2} \eta_{x x x}+\rho_{6} \eta \eta_{x} \eta_{x x}+\rho_{7} \eta_{x}^{3}\right)=0
\end{aligned}
$$

which is an important water wave model, where $\alpha=3 \mathrm{~A} / 2$, $\beta=B / 6, \rho_{1}=-1 / 6, \rho_{6}=5 / 3, \rho_{3}=23 / 6, \rho_{4}=1 / 8$, $\rho_{5}=7 / 18, \rho_{6}=79 / 36$, and $\rho_{7}=45 / 36$. Regarding the $\rho_{1}, \rho_{2}$, 
$\rho_{3}, \rho_{4}, \rho_{5}, \rho_{6}$, and $\rho_{7}$ as free parameters and using the $\tilde{\rho}_{4}$ to replace the $\rho_{4} \alpha^{2}$, (1) becomes the following PDE:

$$
\begin{aligned}
& u_{t}+u_{x}+\alpha u u_{x}+\beta u_{x x x}+\rho_{1} \alpha^{2} u^{2} u_{x} \\
& +\alpha \beta\left(\rho_{2} u u_{x x x}+\rho_{3} u_{x} \eta_{x x}\right)+\tilde{\rho}_{4} \alpha u^{3} u_{x} \\
& +\alpha^{2} \beta\left(\rho_{5} u^{2} u_{x x x}+\rho_{6} u u_{x} u_{x x}+\rho_{7} \eta_{x}^{3}\right)=0
\end{aligned}
$$

which is given by Tzirtzilakis et al. in [18]. They called it highorder wave equation of $\mathrm{KdV}$ type. Just as Tzirtzilakis et al. [18] said, these two equations are both water wave equations of $\mathrm{KdV}$ type, which are more physically and practically meaningful.

Assuming that the waves are unidirectional and neglecting terms of $O\left(\alpha^{2}, \beta^{3}, \alpha \beta\right)$, (1) can be reduced to the classical KdV equation:

$$
\eta_{t}+\eta_{x}+\alpha \eta \eta_{x}+\beta \eta_{x x x}=0
$$

In [17], Fokas assumed that $O(\beta)$ is less than $O(\alpha)$. According to this assumption, we easily know that $O\left(\alpha^{2} \beta\right)<O\left(\alpha^{3}\right)$ and $O\left(\alpha^{2} \beta\right)<O(\alpha \beta)$. Neglecting two high-order infinitesimal terms of $O\left(\alpha^{3}, \alpha^{2} \beta\right)$, (1) can be reduced to another high-order wave equation of $\mathrm{KdV}$ type $[18,19]$ as follows:

$$
\begin{gathered}
\eta_{t}+\eta_{x}+\alpha \eta \eta_{x}+\beta \eta_{x x x}+\rho_{1} \alpha^{2} \eta^{2} \eta_{x} \\
+\alpha \beta\left(\rho_{2} \eta \eta_{x x x}+\rho_{3} \eta_{x} \eta_{x x}\right)=0 .
\end{gathered}
$$

Equation (4) is a special case of (1) for $\rho_{4}=\rho_{5}=\rho_{6}=\rho_{7}=0$. Equation (4) was first derived in [20] by using the method of bi-Hamiltonian systems, whose Lax pair was also given in [21].

If only we neglect the highest-order infinitesimal term of $O\left(\alpha^{2} \beta\right)$, then (1) can be reduced to a new generalized $\mathrm{KdV}$ equation as follows:

$$
\begin{aligned}
\eta_{t} & +\eta_{x}+\alpha \eta \eta_{x}+\beta \eta_{x x x}+\rho_{1} \alpha^{2} \eta^{2} \eta_{x} \\
& +\alpha \beta\left(\rho_{2} \eta \eta_{x x x}+\rho_{3} \eta_{x} \eta_{x x}\right)+\rho_{4} \alpha^{3} \eta^{3} \eta_{x}=0 .
\end{aligned}
$$

In fact, (5) is another special case of (1) for $\rho_{5}=\rho_{6}=$ $\rho_{7}=0$. It is also third-order approximate equation of $\mathrm{KdV}$ type. Of course, on describing dynamical behaviors of water waves, (4) is only a rough approximative model of (1) compared with (5); that is, the precision of model (5) is better than that of model (4) on describing dynamical behaviors of water waves. In other words, the model (5) exhibits much richer phenomenology than the model (4). Therefore, the investigation of exact traveling wave solutions for (5) is more practically meaningful than that of (4).

Equation (1) is studied by many researchers and some useful results are obtained when $\rho_{i}$ takes special values. However, by using the current methods, we cannot obtain exact solutions of (1) in universal conditions. Therefore, the investigation of exact solutions of (5) is necessary and important. Equation (5) is perhaps not integrable. But it would be interesting to check its asymptotic integrability [22]. Equation (5) is studied by Wu et al. in [23] using the integral bifurcation method and some exact solutions in parameter form are given. In this paper, regarding the $\rho_{i}(i=1,2,3,4)$ as free parameters and by using the extended F-expansion method [12], we will investigate exact traveling wave solutions of (5).

The organization of the paper is as follows. In Section 2, a brief description of the extended F-expansion for finding traveling wave solutions of nonlinear equations is given. In Section 3, we will study (5) by the extended F-expansion methods. Finally conclusions are given in Section 4 .

\section{Description of the Extended F-Expansion Methods}

Based on F-expansion method, the main procedures of the extended F-expansion method are as follows [12].

Step 1. Consider a general nonlinear PDE in the form

$$
F\left(u, u_{x}, u_{t}, u_{x x}, u_{x t}, \ldots\right)=0
$$

Using $u(x, t)=U(\xi), \xi=x-c t$, we can rewrite (6) as the following nonlinear ODE:

$$
F\left(U, U^{\prime}, U^{\prime \prime}, \cdots\right)=0
$$

where the prime denotes differentiation with respect to $\xi$.

Step 2. Suppose that the solution of ODE (7) can be written as follows:

$$
U(\xi)=A_{0}+\sum_{i=1}^{n}\left(A_{i} F^{i}(\xi)+B_{i} F^{-i}(\xi)\right),
$$

where $A_{i}, B_{i}(i=1,2, \ldots n)$ are constants to be determined later, $n$ is a positive integer that is given by the homogeneous balance principle, and $F(\xi)$ satisfies the following equation:

$$
\left(F^{\prime}(\xi)\right)^{2}=h_{0}+h_{1} F(\xi)+h_{2} F^{2}(\xi)+h_{3} F^{3}(\xi)+h_{4} F^{4}(\xi),
$$

where $h_{0}, h_{1}, h_{2}, h_{3}$, and $h_{4}$ are constant.

Step 3. Substituting (8) along with (9) into (7) and then setting all the coefficients of $F^{j}(\xi)(j=0,1,2, \ldots$,$) of the$ resulting system's numerator to zero yield a set of overdetermined nonlinear algebraic equations for $A_{0}, A_{i}$, and $B_{i}(i=$ $1,2, \ldots, n)$.

Step 4. Assuming that the constants $A_{0}, A_{i}$, and $B_{i}(i=$ $1,2, \ldots n)$ can be obtained by solving the algebraic equations in Step 3 and then substituting these constants and the solutions of (9), depending on the special conditions chosen for the $h_{0}, h_{1}, h_{2}, h_{3}$, and $h_{4}$, we can obtain the explicit solutions of (6) immediately. 


\section{Exact Solutions of (5)}

Making a transformation $\eta(t, x)=\phi(\xi)$ with $\xi=x-c t$, (5) can be reduced to the following ODE:

$$
\begin{aligned}
-c \phi^{\prime} & +\phi^{\prime}+\alpha \phi \phi^{\prime}+\beta \phi^{\prime \prime \prime}+\rho_{1} \alpha^{2} \phi^{2} \phi^{\prime} \\
& +\alpha \beta\left(\rho_{2} \phi \phi^{\prime \prime \prime}+\rho_{3} \phi^{\prime} \phi^{\prime \prime}\right)+\rho_{4} \alpha^{3} \phi^{3} \phi^{\prime}=0,
\end{aligned}
$$

where $c$ is wave velocity which moves along the direction of $x$-axis and $c \neq 0$. Integrating (10) once and setting the integral constant as $R$ yield

$$
\begin{aligned}
& (1-c) \phi+\frac{1}{2} \alpha \phi^{2}+\beta \phi^{\prime \prime}+\frac{1}{3} \rho_{1} \alpha^{2} \phi^{3} \\
& +\alpha \beta\left[\rho_{2} \phi \phi^{\prime \prime}+\frac{1}{2}\left(\rho_{3}-\rho_{2}\right) \phi^{\prime 2}\right] \\
& +\frac{1}{4} \rho_{4} \alpha^{3} \phi^{4}+R=0 .
\end{aligned}
$$

Suppose that (11) owns the solutions in the form

$$
\phi(\xi)=A_{0}+A_{1} F(\xi)+\frac{B_{1}}{F(\xi)} .
$$

Substituting (12) and (9) into (11) and then setting all the coefficients of $F^{k}(k=-5, \ldots, 5)$ of the resulting system to zero, we can obtain the following results.

3.1. Case of $h_{1}=h_{3}=0$. In this situation, we have the following cases.

Case 1. Consider

$$
\begin{gathered}
A_{0}=-\frac{2 \beta h_{2}}{\alpha}, \quad A_{1}= \pm \frac{2 \beta \sqrt{-6 h_{2} h_{4}}}{\alpha}, \\
B_{1}=0, \quad \rho_{1}=\frac{\rho_{3}}{2}+\rho_{2}+\frac{1}{4 \beta h_{2}}, \\
\rho_{4}=\frac{\rho_{3}+3 \rho_{2}}{12 \beta h_{2}}, \quad c=\frac{4}{3} \beta^{2} h_{2}^{2} \rho_{3}+1,
\end{gathered}
$$

where $h_{2} \neq 0$ and $h_{0}$ and $h_{4}$ are arbitrary constants.

Case 2. Consider

$$
\begin{gathered}
A_{0}=-\frac{2 \beta h_{2}}{\alpha}, \quad A_{1}=0, \\
B_{1}= \pm \frac{2 \beta \sqrt{-6 h_{0} h_{2}}}{\alpha}, \quad \rho_{1}=\frac{\rho_{3}}{2}+\rho_{2}+\frac{1}{4 \beta h_{2}}, \\
\rho_{4}=\frac{\rho_{3}+3 \rho_{2}}{12 \beta h_{2}}, \quad c=\frac{4}{3} \beta^{2} h_{2}^{2} \rho_{3}+1,
\end{gathered}
$$

where $h_{2} \neq 0$ and $h_{0}$ and $h_{4}$ are arbitrary constants.
Case 3. Also

$$
\begin{gathered}
A_{0}=\frac{\alpha B_{1}^{2}}{12 \beta h_{0}}, \quad A_{1}=w B_{1}, \\
\rho_{1}=-\frac{6 \beta h_{0}}{\alpha^{2} B_{1}^{2}}-\frac{1}{2} \rho_{3}, \quad \rho_{2}=-\rho_{3}, \quad \rho_{4}=\frac{m}{4 \beta \rho_{3} h_{0}} \alpha^{2} B_{1}^{2}, \\
c=-\frac{\rho_{3} B_{1}^{4} \alpha^{4}}{864 \beta^{2} h_{0}^{2}}+\frac{\alpha^{2} B_{1}^{2}}{24 \beta h_{0}}+1-6 \beta h_{0} w+\frac{1}{2} w B_{1}^{2} \rho_{3} \alpha^{2} \\
+\beta h_{2}-\frac{B_{1}^{2} \rho_{3} \alpha^{2} h_{2}}{12 h_{0}},
\end{gathered}
$$

where $w= \pm \sqrt{h_{4} / h_{0}}$, and $h_{0} \neq 0, h_{2}$ and $h_{4}$ are arbitrary constants.

Substituting (13)-(15) into (12), we obtain, respectively, the following formal solution of (5):

$$
\eta(x, t)=\frac{2 \beta}{\alpha}\left( \pm \sqrt{-6 h_{2} h_{4}} F(\xi)-h_{2}\right),
$$

where $\xi=x-\left((4 / 3) \beta^{2} h_{2}^{2} \rho_{3}+1\right) t, \rho_{1}=\rho_{3} / 2+\rho_{2}+1 / 4 \beta h_{2}$, and $\rho_{4}=\left(\rho_{3}+3 \rho_{2}\right) / 12 \beta h_{2}$.

Moreover,

$$
\eta(x, t)=\frac{2 \beta}{\alpha}\left( \pm \frac{\sqrt{-6 h_{0} h_{2}}}{F(\xi)}-h_{2}\right),
$$

where $\xi=x-\left((4 / 3) \beta^{2} h_{2}^{2} \rho_{3}+1\right) t, \rho_{1}=\rho_{3} / 2+\rho_{2}+1 / 4 \beta h_{2}$, and $\rho_{4}=\left(\rho_{3}+3 \rho_{2}\right) / 12 \beta h_{2}$.

We have

$$
\eta(x, t)=\frac{\alpha B_{1}^{2}}{12 \beta h_{0}} \pm \sqrt{\frac{h_{4}}{h_{0}}} B_{1} F(\xi)+\frac{B_{1}}{F(\xi)},
$$

where $\xi=x-c t, c$ is determined in Case $3, \rho_{1}=-\left(6 \beta h_{0} /\right.$ $\left.\alpha^{2} B_{1}{ }^{2}\right)-(1 / 2) \rho_{3}, \rho_{2}=-\rho_{3}$, and $\rho_{4}=4 \beta \rho_{3} h_{0} / \alpha^{2} B_{1}{ }^{2}$.

When $h_{1}=h_{3}=0$, the general elliptic equation (9) is reduced to the auxiliary ordinary equation:

$$
F^{\prime}(\xi)^{2}=h_{0}+h_{2} F^{2}(\xi)+h_{4} F^{4}(\xi) \text {. }
$$

The solutions of (19) are given in Table 1. Combining (16)(18) with Table 1, many exact solutions of (5) can be obtained. For simplicity, we just give out one case in Table 1, and the other cases can be discussed similarly.

When $h_{0}=1, h_{2}=-\left(m^{2}+1\right)$, and $h_{4}=m^{2}$, the solution of $(16)$ is $F(\xi)=\operatorname{sn}(\xi, m)$ or $F(\xi)=\operatorname{cd}(\xi, m)$. Substituting them into (17)-(19), we can obtain the following solutions of (5).

From (16), one has

$$
\begin{aligned}
& \eta(x, t)=\frac{2 \beta}{\alpha}\left(m^{2}+1 \pm m \sqrt{6\left(m^{2}+1\right)} \operatorname{sn}(\xi, m)\right) \\
& \eta(x, t)=\frac{2 \beta}{\alpha}\left(m^{2}+1 \pm m \sqrt{6\left(m^{2}+1\right)} \operatorname{cd}(\xi, m)\right)
\end{aligned}
$$


TABLE 1: Solutions of $F(\xi)$ in $F^{\prime 2}=h_{0}+h_{2} F^{2}+h_{4} F^{4}$.

\begin{tabular}{|c|c|c|c|c|}
\hline Case & $h_{0}$ & $h_{2}$ & $h_{4}$ & $F(\xi)$ \\
\hline 1 & 1 & $-\left(m^{2}+1\right)$ & $m^{2}$ & $\overline{\operatorname{sn}(\xi), \operatorname{cd}(\xi)}$ \\
\hline 2 & $1-m^{2}$ & $m^{2}-1$ & $-m^{2}$ & $\operatorname{cn}(\xi)$ \\
\hline 3 & $m^{2}-1$ & $2-m^{2}$ & -1 & $\operatorname{dn}(\xi)$ \\
\hline 4 & $m^{2}$ & $-\left(m^{2}+1\right)$ & 1 & $\mathrm{~ns}(\xi), \mathrm{dc}(\xi)$ \\
\hline 5 & $-m^{2}$ & $2 m^{2}-1$ & $1-m^{2}$ & $\mathrm{nc}(\xi)$ \\
\hline 6 & -1 & $2-m^{2}$ & $m^{2}-1$ & $\operatorname{nd}(\xi)$ \\
\hline 7 & 1 & $2-m^{2}$ & $1-m^{2}$ & $\operatorname{sc}(\xi)$ \\
\hline 8 & 1 & $2 m^{2}-1$ & $-m^{2}\left(1-m^{2}\right)$ & $\operatorname{sd}(\xi)$ \\
\hline 9 & $1-m^{2}$ & $2-m^{2}$ & 1 & $\operatorname{cs}(\xi)$ \\
\hline 10 & $-m^{2}\left(1-m^{2}\right)$ & $2 m^{2}-1$ & 1 & $\operatorname{sd}(\xi)$ \\
\hline 11 & $\frac{1}{4}$ & $\frac{1-2 m^{2}}{2}$ & $\frac{1}{4}$ & $\mathrm{~ns}(\xi) \pm \mathrm{cs}(\xi)$ \\
\hline 12 & $\frac{1-m^{2}}{4}$ & $\frac{1+m^{2}}{2}$ & $\frac{1-m^{2}}{4}$ & $\mathrm{nc}(\xi) \pm \mathrm{sc}(\xi)$ \\
\hline 13 & $\frac{m^{2}}{4}$ & $\frac{m^{2}-2}{2}$ & $\frac{1}{4}$ & $\mathrm{~ns}(\xi) \pm \mathrm{ds}(\xi)$ \\
\hline 14 & $\frac{m^{2}}{4}$ & $\frac{m^{2}-2}{2}$ & $\frac{m^{2}}{4}$ & $\operatorname{sn}(\xi) \pm i \operatorname{cn}(\xi)$ \\
\hline
\end{tabular}

where $\xi=x-\left((4 / 3) \beta^{2} \rho_{3}\left(m^{2}+1\right)^{2}+1\right) t, \rho_{1}=\rho_{3} / 2+\rho_{2}-$ $\left(1 / 4 \beta\left(m^{2}+1\right)\right)$, and $\rho_{4}=-\left(\rho_{3}+3 \rho_{2}\right) / 12 \beta\left(m^{2}+1\right)$.

When $m \rightarrow 1, \operatorname{sn}(\xi, m) \rightarrow \tanh (\xi),(20)$ becomes

$$
\eta(x, t)=\frac{4 \beta}{\alpha}(1 \pm \sqrt{3} \tanh (\xi))
$$

where $\xi=x-\left((16 / 3) \beta^{2} \rho_{3}+1\right) t, \rho_{1}=\rho_{3} / 2+\rho_{2}-1 / 8 \beta$, and $\rho_{4}=-\left(\rho_{3}+3 \rho_{2}\right) / 24 \beta$.

From (17), we have

$$
\begin{aligned}
& \eta(x, t)=\frac{2 \beta}{\alpha}\left(m^{2}+1 \pm \sqrt{6\left(m^{2}+1\right)} \mathrm{ns}(\xi, m)\right), \\
& \eta(x, t)=\frac{2 \beta}{\alpha}\left(m^{2}+1 \pm \sqrt{6\left(m^{2}+1\right)} \mathrm{dc}(\xi, m)\right),
\end{aligned}
$$

where $\xi=x-\left((4 / 3) \beta^{2} \rho_{3}\left(m^{2}+1\right)^{2}+1\right) t, \rho_{1}=\rho_{3} / 2+\rho_{2}-$ $\left(1 / 4 \beta\left(m^{2}+1\right)\right), \rho_{4}=-\left(\rho_{3}+3 \rho_{2}\right) / 12 \beta\left(m^{2}+1\right)$.

When $m \rightarrow 1$, ns $(\xi, m) \rightarrow \operatorname{coth}(\xi),(23)$ becomes

$$
\eta(x, t)=\frac{4 \beta}{\alpha}(1 \pm \sqrt{3} \operatorname{coth}(\xi))
$$

where $\xi=x-\left((16 / 3) \beta^{2} \rho_{3}+1\right) t, \rho_{1}=\rho_{3} / 2+\rho_{2}-1 / 8 \beta$, and $\rho_{4}=-\left(\rho_{3}+3 \rho_{2}\right) / 24 \beta$.
When $m \rightarrow 0$, ns $(\xi, m) \rightarrow \csc (\xi)$, and $\mathrm{dc}(\xi, m) \rightarrow \sec (\xi)$, (23) and (24) become

$$
\begin{aligned}
& \eta(x, t)=\frac{2 \beta}{\alpha}(1 \pm \sqrt{6} \csc (\xi)), \\
& \eta(x, t)=\frac{2 \beta}{\alpha}(1 \pm \sqrt{6} \sec (\xi)),
\end{aligned}
$$

where $\xi=x-\left((4 / 3) \beta^{2} \rho_{3}+1\right) t, \rho_{1}=\rho_{3} / 2+\rho_{2}-1 / 4 \beta$, and $\rho_{4}=-\left(\rho_{3}+3 \rho_{2}\right) / 12 \beta$.

From (18), we have

$$
\begin{aligned}
& \eta(x, t)=\frac{\alpha B_{1}^{2}}{12 \beta} \pm m B_{1} \operatorname{sn}(\xi, m)+\frac{B_{1}}{\operatorname{sn}(\xi, m)}, \\
& \eta(x, t)=\frac{\alpha B_{1}^{2}}{12 \beta} \pm m B_{1} \mathrm{~cd}(\xi, m)+\frac{B_{1}}{\mathrm{~cd}(\xi, m)},
\end{aligned}
$$

where $\xi=x-c t, c$ is determined in Case $3, \rho_{1}=-(6 \beta /$ $\left.\alpha^{2} B_{1}^{2}\right)-(1 / 2) \rho_{3}, \rho_{2}=-\rho_{3}$, and $\rho_{4}=4 \beta \rho_{3} / \alpha^{2} B_{1}^{2}$.

When $m \rightarrow 1, \operatorname{sn}(\xi, m) \rightarrow \tanh (\xi),(27)$ becomes

$$
\eta(x, t)=\frac{\alpha B_{1}^{2}}{12 \beta} \pm B_{1} \tanh (\xi)+B_{1} \operatorname{coth}(\xi),
$$


where $\xi=x-c t, \rho_{1}=-\left(6 \beta / \alpha^{2} B_{1}^{2}\right)-(1 / 2) \rho_{3}, \rho_{2}=-\rho_{3}$, and $\rho_{4}=4 \beta \rho_{3} / \alpha^{2} B_{1}^{2}$.

When $m \rightarrow 0, \operatorname{sn}(\xi, m) \rightarrow \sin (\xi)$, and $\operatorname{dc}(\xi, m) \rightarrow \sec (\xi)$, (27) and (28) become

$$
\begin{aligned}
& \eta(x, t)=\frac{\alpha B_{1}^{2}}{12 \beta} \pm B_{1} \csc (\xi), \\
& \eta(x, t)=\frac{\alpha B_{1}^{2}}{12 \beta} \pm B_{1} \sec (\xi),
\end{aligned}
$$

where $\xi=x-c t, \rho_{1}=-6 \beta / \alpha^{2} B_{1}^{2}-(1 / 2) \rho_{3}, \rho_{2}=-\rho_{3}$, and $\rho_{4}=$ $4 \beta \rho_{3} / \alpha^{2} B_{1}^{2}$.

3.2. Case of $h_{0}=h_{1}=0$. In this situation, we have

$$
\begin{gathered}
A_{0}=\frac{\beta\left(h_{3} \sqrt{w}-8 h_{4} h_{2}+3 h_{3}^{2}\right)}{4 \alpha h_{4}}, \\
A_{1}=\frac{\beta \sqrt{w}}{\alpha}, \quad B_{1}=0, \\
\rho_{1}=\rho_{2}+\frac{\rho_{3}}{2}-\frac{6 h_{4}}{\beta w}, \quad \rho_{4}=-\frac{2 h_{4}\left(3 \rho_{2}+\rho_{3}\right)}{\beta w}, \\
c=1+\frac{4}{3} \rho_{3} \beta^{2} h_{2}^{2}-\frac{\rho_{3} \beta^{2} h_{2} h_{3}\left(4 h_{3}+\sqrt{w}\right)}{4 h_{4}} \\
+\frac{\rho_{3} \beta^{2} h_{3}^{3}\left(3 h_{3}+\sqrt{w}\right)}{16 h_{4}^{2}},
\end{gathered}
$$

where $w=9 h_{3}^{2}-24 h_{2} h_{4}$ and $h_{2}, h_{3}, h_{4}, \rho_{2}$, and $\rho_{3}$ are arbitrary constants.

Substituting (3.2) into (12), we obtain the following formal solution of (5):

$$
\eta(x, t)=\frac{\beta\left(h_{3} \sqrt{w}-8 h_{4} h_{2}+3 h_{3}^{2}\right)}{4 \alpha h_{4}}+\frac{\sqrt{w} \beta}{\alpha} F(\xi),
$$

where $\xi=x-c t$ and $c, \rho_{1}$, and $\rho_{4}$ are determined in (3.2).

When $h_{0}=h_{1}=0$, the general elliptic equation (9) is reduced to the auxiliary ordinary equation:

\begin{tabular}{|c|c|c|}
\hline Case & $h_{4}, \Delta$ & $F(\xi)$ \\
\hline \multirow{2}{*}{1} & \multirow{2}{*}{$h_{2}>0$} & $-h_{2} h_{3} \operatorname{sech}^{2}\left(\left(\sqrt{h_{2}} / 2\right) \xi\right)$ \\
\hline & & $\overline{h_{3}^{2}-h_{2} h_{4}\left(1+\varepsilon \tan h\left(\left(\sqrt{h_{2}} / 2\right) \xi\right)\right)}$ \\
\hline \multirow{2}{*}{2} & \multirow{2}{*}{$h_{2}>0$} & $-h_{2} h_{3} \operatorname{csch}^{2}\left(\left(\sqrt{h_{2}} / 2\right) \xi\right)$ \\
\hline & & $\overline{h_{3}^{2}-h_{2} h_{4}\left(1+\varepsilon \cot h\left(\left(\sqrt{h_{2}} / 2\right) \xi\right)\right)}$ \\
\hline \multirow{2}{*}{3} & \multirow{2}{*}{$h_{2}>0, \Delta>0$} & $2 h_{2} \sec h\left(\sqrt{h_{2}} \xi\right)$ \\
\hline & & $\varepsilon \sqrt{\Delta}-h_{3} \operatorname{sech}\left(\sqrt{h_{2}} \xi\right)$ \\
\hline \multirow{2}{*}{4} & \multirow{2}{*}{$h_{2}<0, \Delta>0$} & $2 h_{2} \sec \left(\sqrt{-h_{2}} \xi\right)$ \\
\hline & & $\varepsilon \sqrt{\Delta}-h_{3} \sec \left(\sqrt{-h_{2}} \xi\right)$ \\
\hline \multirow{2}{*}{5} & \multirow{2}{*}{$h_{2}>0, \Delta<0$} & $2 h_{2} \operatorname{csch}\left(\sqrt{h_{2}} \xi\right)$ \\
\hline & & $\overline{\varepsilon \sqrt{-\Delta}-h_{3} \operatorname{csch}\left(\sqrt{h_{2}} \xi\right)}$ \\
\hline \multirow{2}{*}{6} & \multirow{2}{*}{$h_{2}<0, \Delta>0$} & $2 h_{2} \csc \left(\sqrt{-h_{2}} \xi\right)$ \\
\hline & & $\overline{\varepsilon \sqrt{\Delta}-h_{3} \csc \left(\sqrt{-h_{2}} \xi\right)}$ \\
\hline \multirow{2}{*}{7} & \multirow{2}{*}{$h_{2}>0, h_{4}>0$} & $-h_{2} \sec h^{2}\left(\left(\sqrt{h_{2}} / 2\right) \xi\right)$ \\
\hline & & $\overline{h_{3}+2 \varepsilon \sqrt{h_{2} h_{4}} \tan h\left(\left(\sqrt{h_{2}} / 2\right) \xi\right)}$ \\
\hline \multirow{2}{*}{8} & \multirow{2}{*}{$h_{2}<0, h_{4}>0$} & $-h_{2} \sec ^{2}\left(\left(\sqrt{-h_{2}} / 2\right) \xi\right)$ \\
\hline & & $\overline{h_{3}+2 \varepsilon \sqrt{-h_{2} h_{4}} \tan \left(\left(\sqrt{-h_{2}} / 2\right) \xi\right)}$ \\
\hline \multirow{2}{*}{9} & \multirow{2}{*}{$h_{2}>0, h_{4}>0$} & $h_{2} \operatorname{csch}^{2}\left(\left(\sqrt{h_{2}} / 2\right) \xi\right)$ \\
\hline & & $\overline{h_{3}+2 \varepsilon \sqrt{h_{2} h_{4}} \cot h\left(\left(\sqrt{h_{2}} / 2\right) \xi\right)}$ \\
\hline \multirow{2}{*}{10} & \multirow{2}{*}{$h_{2}<0, h_{4}>0$} & $-h_{2} \csc ^{2}\left(\left(\sqrt{-h_{2}} / 2\right) \xi\right)$ \\
\hline & & $\overline{h_{3}+2 \varepsilon \sqrt{-h_{2} h_{4}} \cot \left(\left(\sqrt{-h_{2}} / 2\right) \xi\right)}$ \\
\hline 11 & $h_{2}>0, \Delta=0$ & $\frac{-h_{2}}{h_{3}}\left(1+\varepsilon \tan h\left(\frac{\sqrt{h_{2}}}{2}\right) \xi\right)$ \\
\hline 12 & $h_{2}>0, \Delta=0$ & $\frac{-h_{2}}{h_{3}}\left(1+\varepsilon \cot h\left(\frac{\sqrt{h_{2}}}{2}\right) \xi\right)$ \\
\hline \multirow{2}{*}{13} & \multirow{2}{*}{$h_{2}>0$} & $4 h_{2} \exp \left(\varepsilon \sqrt{h_{2}} \xi\right)$ \\
\hline & & $\overline{\left(\exp \left(\varepsilon \sqrt{h_{2}} \xi\right)-h_{3}\right)^{2}-4 h_{2} h_{4}}$ \\
\hline \multirow{2}{*}{14} & \multirow{2}{*}{$h_{2}>0, h_{3}=0$} & $4 h_{2} \exp \left(\varepsilon \sqrt{h_{2}} \xi\right)$ \\
\hline & & $1-4 h_{2} h_{4} \exp \left(2 \varepsilon \sqrt{h_{2}} \xi\right)$ \\
\hline
\end{tabular}

$$
F^{\prime}(\xi)^{2}=h_{2} F^{2}(\xi)+h_{3} F^{3}(\xi)+h_{4} F^{4}(\xi) .
$$

The solutions of (33) are given in Table 2.

Combining (32) with Table 2, many exact solutions of (5) can be obtained. The following is an example.

If $h_{2}>0$, solution of (33) is
TABLE 2: Solutions of (33) with $\Delta=h_{3}^{2}-4 h_{2} h_{4}, \varepsilon= \pm 1$.

$$
F(\xi)=\frac{-h_{2} h_{3} \operatorname{sech}^{2}\left(\left(\sqrt{h_{2}} / 2\right) \xi\right)}{h_{3}^{2}-h_{2} h_{4}\left(1+\varepsilon \tanh \left(\left(\sqrt{h_{2}} / 2\right) \xi\right)\right)} .
$$

Therefore, solution of (4) is

$$
\begin{aligned}
\eta(x, t)= & \frac{\beta\left(h_{3} \sqrt{w}-8 h_{2} h_{4}+3 h_{3}^{2}\right)}{4 \alpha h_{4}} \\
& +\frac{\beta \sqrt{w}}{\alpha} \frac{-h_{2} h_{3} \operatorname{sech}^{2}\left(\left(\sqrt{h_{2}} / 2\right) \xi\right)}{h_{3}^{2}-h_{2} h_{4}\left(1+\varepsilon \tanh \left(\left(\sqrt{h_{2}} / 2\right) \xi\right)\right)},
\end{aligned}
$$

where $\xi=x-c t, w=9 h_{3}^{2}-24 h_{2} h_{4}$, and $c, \rho_{1}$, and $\rho_{4}$ are determined by (3.2). 
3.3. Case of $h_{0} \neq 0, h_{1} \neq 0, h_{2} \neq 0, h_{3} \neq 0$, and $h_{4} \neq 0$. In this case, there exist three parameters $r, p$, and $q$ such that

$$
\begin{aligned}
\left(F^{\prime}(\xi)\right)^{2} & =h_{0}+h_{1} F(\xi)+h_{2} F^{2}(\xi)+h_{3} F^{3}(\xi)+h_{4} F^{4}(\xi) \\
& =\left(r+p F(\xi)+q F^{2}(\xi)\right)^{2}
\end{aligned}
$$

Equation (36) is satisfied only if the following relations hold:

$$
\begin{gathered}
h_{0}=r^{2}, \quad h_{1}=2 r p, \\
h_{2}=2 r q+p^{2}, \quad h_{3}=2 p q, \quad h_{4}=q^{2} .
\end{gathered}
$$

Equation (36) is the general Riccati equation. The solutions of (36) are listed in [12]. There are 24 group solutions named $\phi_{i}^{I}(i=1,2, \ldots 24)$, which we do not list for simplicity.

Substituting (36) and (9) into (11) and then setting all the coefficients of $F^{k}(k=-4, \ldots, 4)$ of the resulting system to zero, we can obtain the following results.

Case 1. Consider

$$
\begin{gathered}
A_{0}=\frac{\beta\left(w p+2 p^{2}-8 q r\right)}{2 \alpha}, \quad A_{1}=0, \\
B_{1}=\frac{w r \beta}{\alpha}, \quad c=1+\frac{1}{3} \beta^{2} \rho_{3}\left(p^{2}-4 q r\right)^{2}, \\
\rho_{1}=\frac{\rho_{3}}{2}+\rho_{2}-\frac{1}{2 \beta\left(p^{2}-4 q r\right)}, \\
\rho_{2}=\rho_{2}, \quad \rho_{3}=\rho_{3}, \\
\rho_{4}=-\frac{\rho_{3}+3 \rho_{2}}{6 \beta\left(p^{2}-4 q r\right)},
\end{gathered}
$$

where $w= \pm 2 \sqrt{3} \sqrt{p^{2}-4 q r}$ and $p^{2}-4 q r>0$.

Case 2. Consider

$$
\begin{gathered}
A_{0}=\frac{\beta\left(w p+2 p^{2}-8 q r\right)}{2 \alpha}, \quad A_{1}=\frac{w r \beta}{\alpha}, \\
B_{1}=0, \quad c=1+\frac{1}{3} \beta^{2} \rho_{3}\left(p^{2}-4 q r\right)^{2}, \\
\rho_{1}=\frac{\rho_{3}}{2}+\rho_{2}-\frac{1}{2 \beta\left(p^{2}-4 q r\right)}, \\
\rho_{2}=\rho_{2}, \quad \rho_{3}=\rho_{3}, \\
\rho_{4}=-\frac{\rho_{3}+3 \rho_{2}}{6 \beta\left(p^{2}-4 q r\right)},
\end{gathered}
$$

Case 3. Consider

$$
\begin{gathered}
A_{0}=\frac{\beta\left(\delta p+16 q r+2 p^{2}\right)}{2 \alpha}, \quad A_{1}=\frac{\delta \beta q}{\alpha}, \\
B_{1}=\frac{r \delta \beta}{\alpha}, \quad c=1-\frac{1}{3} \rho_{3} \beta^{2}\left(6 r p \delta q-\left(8 q r+p^{2}\right)^{2}\right), \\
\rho_{1}=\frac{\rho_{3}}{2}+\rho_{2}-\frac{1}{2 \beta\left(8 q r+p^{2}\right)}, \quad \rho_{2}=\rho_{2}, \\
\rho_{3}=\rho_{3}, \quad \rho_{4}=-\frac{\rho_{3}+3 \rho_{2}}{6 \beta\left(8 q r+p^{2}\right)},
\end{gathered}
$$

where $\delta= \pm 2 \sqrt{3} \sqrt{p^{2}+8 q r}$ and $p^{2}+8 q r>0$.

Substituting (38)-(40) into (12), we obtain, respectively, the following formal solution of (5):

$$
\eta(x, t)=\frac{\beta\left(w p+2 p^{2}-8 q r\right)}{2 \alpha}+\frac{w r \beta}{\alpha} F(\xi),
$$

where $\xi=x-\left(1+(1 / 3) \beta^{2} \rho_{3}\left(p^{2}-4 q r\right)^{2}\right) t, \rho_{1}=\left(\rho_{3} / 2\right)+\rho_{2}-$ $1 / 2 \beta\left(p^{2}-4 q r\right)$, and $\rho_{4}=-\left(\rho_{3}+3 \rho_{2}\right) / 6 \beta\left(p^{2}-4 q r\right)$.

Also

$$
\eta(x, t)=\frac{\beta\left(w p+2 p^{2}-8 q r\right)}{2 \alpha}+\frac{w r \beta}{\alpha} \frac{1}{F(\xi)},
$$

where $\xi=x-\left(1+(1 / 3) \beta^{2} \rho_{3}\left(p^{2}-4 q r\right)^{2}\right) t, \rho_{1}=\left(\rho_{3} / 2\right)+\rho_{2}-$ $1 / 2 \beta\left(p^{2}-4 q r\right)$, and $\rho_{4}=-\left(\rho_{3}+3 \rho_{2}\right) / 6 \beta\left(p^{2}-4 q r\right)$.

Moreover,

$$
\eta(x, t)=\frac{\beta\left(\delta p+16 q r+2 p^{2}\right)}{2 \alpha}+\frac{\delta \beta q}{\alpha} F(\xi)+\frac{r \delta \beta}{\alpha} \frac{1}{F(\xi)},
$$

where $\xi=x-\left(1-(1 / 3) \rho_{3} \beta^{2}\left(6 r p \delta q-\left(8 q r+p^{2}\right)^{2}\right)\right) t, \rho_{1}=$ $\left(\rho_{3} / 2\right)+\rho_{2}-1 / 2 \beta\left(8 q r+p^{2}\right)$, and $\rho_{4}=-\left(\rho_{3}+3 \rho_{2}\right) / 6 \beta\left(8 q r+p^{2}\right)$.

Substituting solutions of $(36) \phi_{i}^{I}(i=1,2, \ldots, 24)$ into (41)-(43), we can obtain a lot of solutions of (5). We just give one example.

When $p^{2}-4 p q>0$ and $p q \neq 0, \phi_{1}^{I}=-(1 / 2 q)(p+$ $\left.\sqrt{p^{2}-4 q r} \tanh \left(\left(\sqrt{p^{2}-4 q r} / 2\right) \xi\right)\right)$. Substituting $\phi_{1}^{I}$ into $(41)$ and (42), we have

$$
\begin{aligned}
& \eta(x, t)=\frac{\beta\left(w p+2 p^{2}-8 q r\right)}{2 \alpha}+\frac{w r \beta}{\alpha} \phi_{1}^{I}, \\
& \eta(x, t)=\frac{\beta\left(w p+2 p^{2}-8 q r\right)}{2 \alpha}+\frac{w r \beta}{\alpha} \frac{1}{\phi_{1}^{I}},
\end{aligned}
$$

where $\xi=x-c t$ and $c, w, \rho_{1}$, and $\rho_{4}$ are determined by (38). 
3.4. Case of $h_{0} \neq 0, h_{1} \neq 0, h_{2}=0, h_{3} \neq 0$, and $h_{4} \neq 0$. In this case, there exist three parameters $r, p$, and $q$ such that

$$
\begin{aligned}
\left(F^{\prime}(\xi)\right)^{2} & =h_{0}+h_{1} F(\xi)+h_{3} F^{3}(\xi)+h_{4} F^{4}(\xi) \\
& =\left(r+p F(\xi)+q F^{2}(\xi)\right)^{2} .
\end{aligned}
$$

Equation (45) is satisfied only if the following relations hold:

$$
\begin{array}{ll}
h_{0}=r^{2}, & h_{1}=2 r p, \\
h_{3}=2 p q, & h_{4}=q^{2} .
\end{array}
$$

The following constraint should exist between $r, p$, and $\mathrm{q}$ parameters:

$$
p^{2}=-2 q r, \quad q r<0 .
$$

Therefore, we can discuss the solution of (5) similarly as in the case shown in Section 3.3 under condition (47). Here, we omit it.

\section{Conclusions}

The investigation of the exact solutions of (5) is very important. And (5) is just studied by Wu et al. [23] using the integral bifurcation method. In our work, (5) is studied by extended F-expansion method and some new exact solutions expressed by Jacobi elliptic function, hyperbolic function, and trigonometric function are obtained. We believe that the results we obtained are useful in describing related physical phenomena. The correctness of all the solutions is verified by substituting them into original equation (5). Comparing with [23], it is easy to see that our method is more straightforward and the forms of the solutions obtained in our paper are also more simple. The related results are enriched.

\section{Conflict of Interests}

The authors declare that there is no conflict of interests regarding the publication of this paper.

\section{Acknowledgments}

This research is supported by the Natural Science Foundation of China (no. 11161020 and no. 11361023) and the Natural Science Foundation of Yunnan (2013FZ117).

\section{References}

[1] M. J. Ablowitz and P. A. Clarkson, Solitons, Nonlinear Evolution Equations and Inverse Scattering, Cambridge University Press, New York, NY, USA, 1991.

[2] V. B. Matveev and M. A. Salle, Darboux Transformations and Solitons, Springer, Berlin, Germany, 1991.

[3] R. Hirota, "Exact solutions of the Korteweg-de Vries eauation for multiple collisions of solutions," Physical Review Letters, vol. 27, pp. 1192-1194, 1971.
[4] M. Wang, Y. Zhou, and Z. Li, "Application of a homogeneous balance method to exact solutions of nonlinear equations in mathematical physics," Physics Letters A, vol. 216, pp. 67-75, 1996.

[5] S. Liu, Z. Fu, S. Liu, and Q. Zhao, "Jacobi elliptic function expansion method and periodic wave solutions of nonlinear wave equations," Physics Letters A, vol. 289, no. 1-2, pp. 69-74, 2001.

[6] E. J. Parkes and B. R. Duffy, "An automated tanh-function method for finding solitary wave solutions to non-linear evolution equations," Computer Physics Communications, vol. 98, pp. 288-300, 1996.

[7] J.-H. He and X.-H. Wu, "Exp-function method for nonlinear wave equations," Chaos, Solitons \& Fractals, vol. 30, no. 3, pp. 700-708, 2006.

[8] A. J. Mohamad Jawad, M. D. Petković, and A. Biswas, "Modified simple equation method for nonlinear evolution equations," Applied Mathematics and Computation, vol. 217, no. 2, pp. 869$877,2010$.

[9] E. Fan, "Uniformly constructing a series of explicit exact solutions to nonlinear equations in mathematical physics," Chaos, Solitons and Fractals, vol. 16, no. 5, pp. 819-839, 2003.

[10] M. Wang and X. Li, "Applications of F-expansion to periodic wave solutions for a new Hamiltonian amplitude equation," Chaos, Solitons and Fractals, vol. 24, no. 5, pp. 1257-1268, 2005.

[11] D. Wang and H.-Q. Zhang, "Further improved F-expansion method and new exact solutions of Konopelchenko-Dubrovsky equation," Chaos, Solitons and Fractals, vol. 25, no. 3, pp. 601610, 2005.

[12] E. Yomba, "The extended Fan's sub-equation method and its application to $\mathrm{KdV}-\mathrm{MKdV}$, BKK and variant Boussinesq equations," Physics Letters A, vol. 336, no. 6, pp. 463-476, 2005.

[13] N. A. Kudryashov, "Exact solutions of the generalized Kuramoto-Sivashinsky equation," Physics Letters A, vol. 147, no. 5-6, pp. 287-291, 1990.

[14] N. A. Kudryashov, "On types of nonlinear nonintegrable equations with exact solutions," Physics Letters A, vol. 155, no. 4-5, pp. 269-275, 1991.

[15] N. A. Kudryashov, "Exact solitary waves of the Fisher equation," Physics Letters A, vol. 342, no. 1-2, pp. 99-106, 2005.

[16] N. A. Kudryashov, "Simplest equation method to look for exact solutions of nonlinear differential equations," Chaos, Solitons and Fractals, vol. 24, no. 5, pp. 1217-1231, 2005.

[17] A. S. Fokas, "On a class of physically important integrable equations," Physica D, vol. 87, no. 1-4, pp. 145-150, 1995.

[18] E. Tzirtzilakis, V. Marinakis, C. Apokis, and T. Bountis, "Soliton-like solutions of higher order wave equations of the Korteweg-de Vries type," Journal of Mathematical Physics, vol. 43, no. 12, pp. 6151-6165, 2002.

[19] E. Tzirtzilakis, M. Xenos, V. Marinakis, and T. C. Bountis, "Interactions and stability of solitary waves in shallow water," Chaos, Solitons \& Fractals, vol. 14, no. 1, pp. 87-95, 2002.

[20] B. Fuchssteiner and A. S. Fokas, "Symplectic structures, their Bäcklund transformations and hereditary symmetries," Physica D, vol. 4, no. 1, pp. 47-66, 1981.

[21] R. Camassa and D. D. Holm, "An integrable shallow water equation with peaked solitons," Physical Review Letters, vol. 71, no. 11, pp. 1661-1664, 1993. 
[22] Y. Kodama and A. V. Mikhailov, "Obstacles to asymptotic integrability," in Algebraic Aspects of Integrable Systems, I. M. Gelfand and A. Fokas, Eds., Birkhäuser, Boston, Mass, USA, 1996.

[23] X. Wu, W. Rui, and X. Hong, "A generalized KdV equation of neglecting the highest-order infinitesimal term and its exact traveling wave solutions," Abstract and Applied Analysis, vol. 2013, Article ID 656297, 15 pages, 2013. 


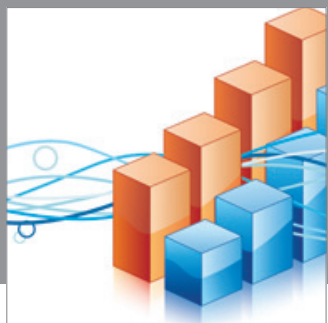

Advances in

Operations Research

mansans

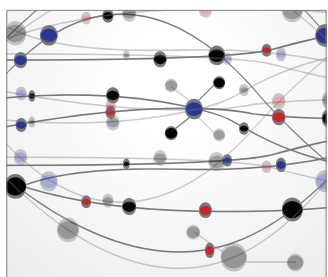

The Scientific World Journal
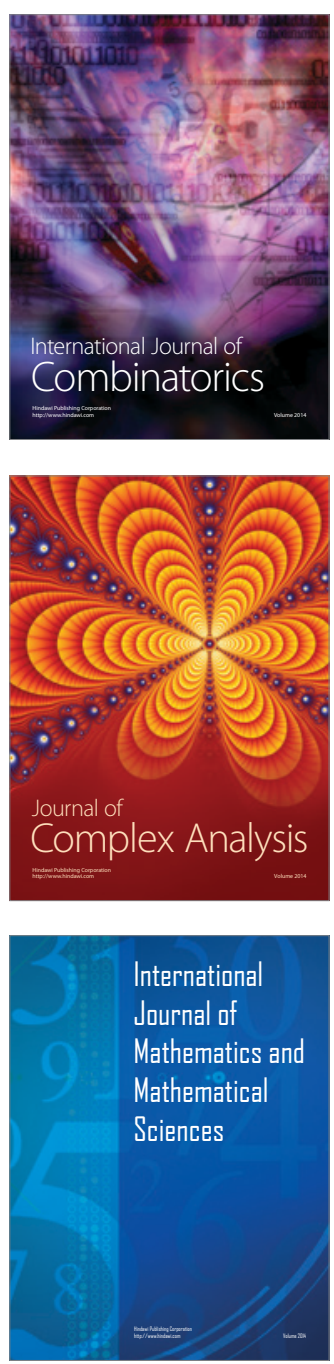
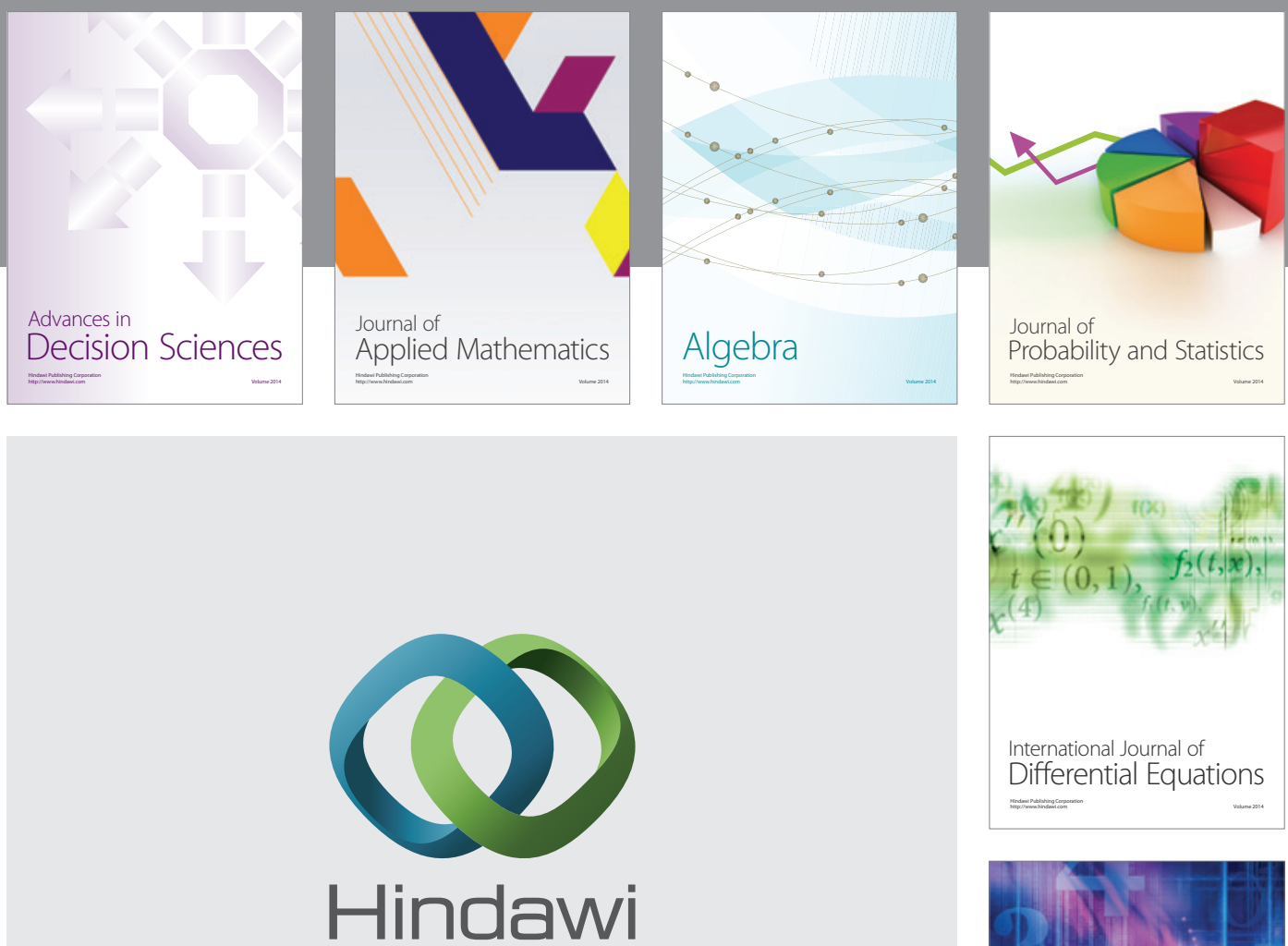

Submit your manuscripts at http://www.hindawi.com
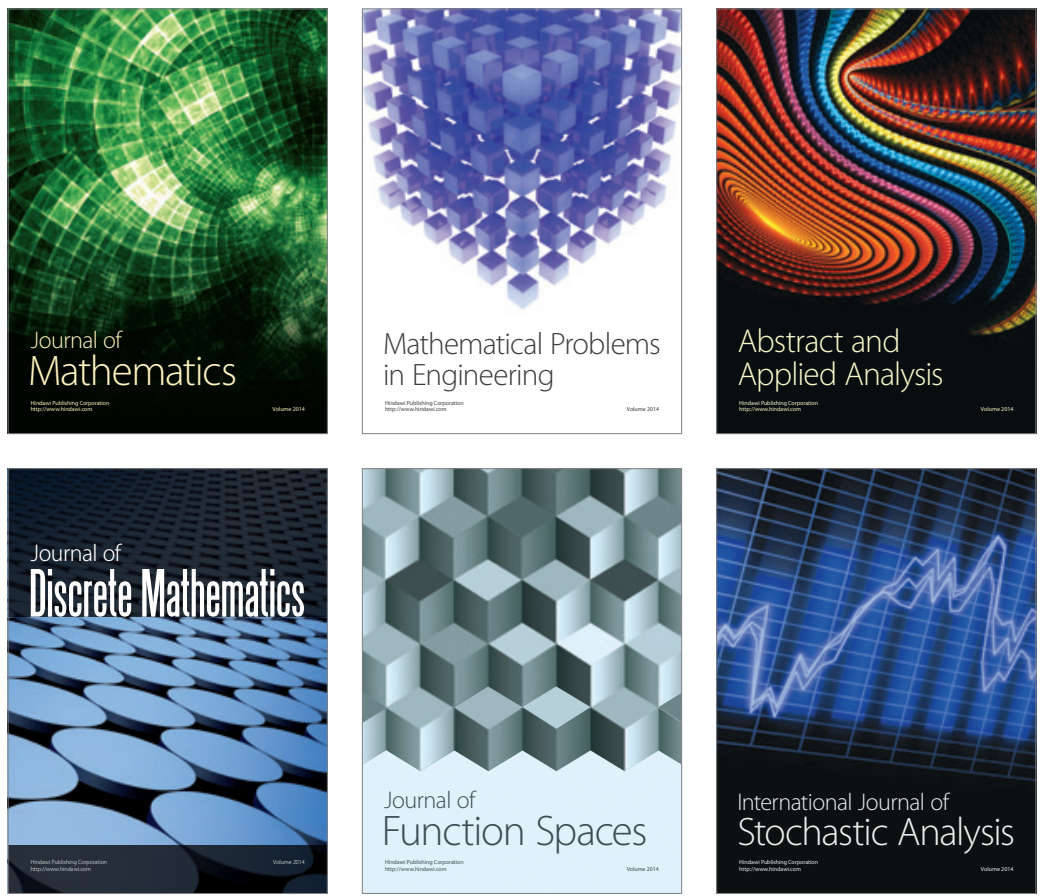

Journal of

Function Spaces

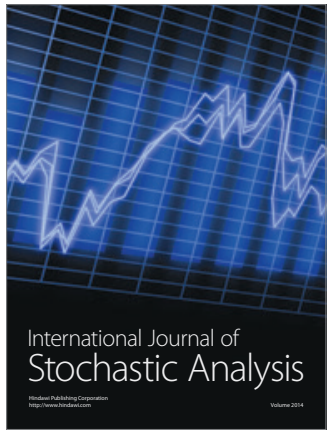

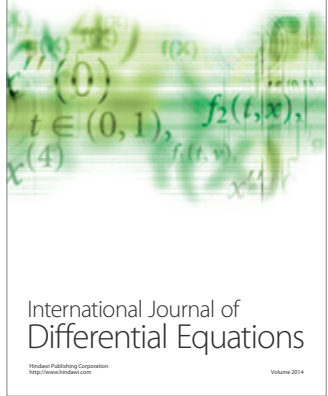
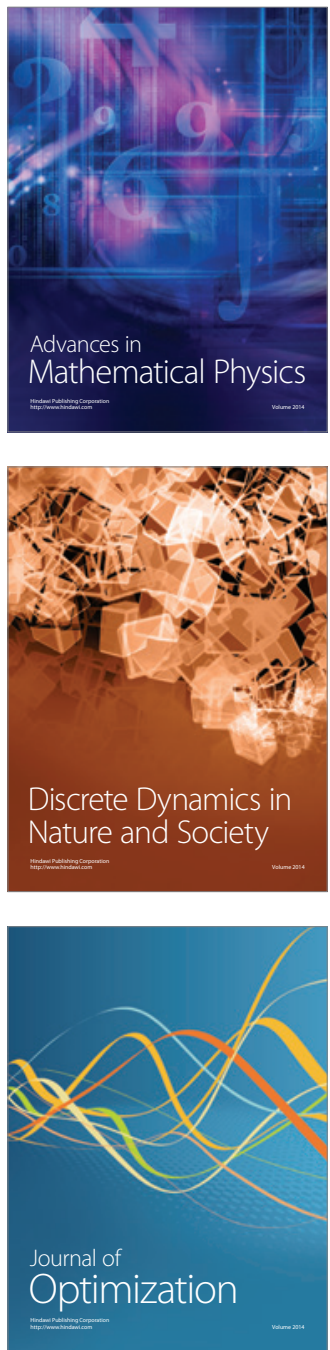